\title{
Norois
}

Environnement, aménagement, société

\section{Conflits d'usage littoraux et intérieurs : une géographie du contentieux de seconde instance dans le département de l'Hérault}

Land use conflicts in coastal zone and further inland: appeal litigation geography in the French department of Hérault

Anne Cadoret, Ségolène Darly, Thierry Kirat et Valérie Lavaud-Letilleul

\section{OpenEdition}

Journals

Édition électronique

URL : http://journals.openedition.org/norois/4328

DOI : $10.4000 /$ norois.4328

ISBN : 978-2-7535-2634-1

ISSN : 1760-8546

Éditeur

Presses universitaires de Rennes

Édition imprimée

Date de publication : 30 décembre 2012

Pagination : $29-46$

ISBN : 978-2-7535-2633-4

ISSN : 0029-182X

Référence électronique

Anne Cadoret, Ségolène Darly, Thierry Kirat et Valérie Lavaud-Letilleul, « Conflits d'usage littoraux et intérieurs : une géographie du contentieux de seconde instance dans le département de l'Hérault », Norois [En ligne], 225 | 2012, mis en ligne le 30 décembre 2014, consulté le 30 avril 2019. URL : http:// journals.openedition.org/norois/4328; DOI : 10.4000/norois.4328 


\title{
Conflits d'usage littoraux et intérieurs : une géographie du contentieux de seconde instance dans le département de l'Hérault
}

\author{
Land use Conflicts in Coastal Zone and Further Inland: \\ Appeal Litigation Geography in the French Department of Hérault
}

Anne Cadoret ${ }^{\mathrm{a} *}$, Ségolène Darly ${ }^{\mathrm{b}}$, Thierry Kiratc, Valérie Lavaud-Letilleul ${ }^{\mathrm{d}}$

\footnotetext{
*Auteur correspondant

a TELEMME UMR 7303 CNRS-Aix-Marseille-Université, 5 rue du Château-de-l'Horloge, 13094 Aix-en-Provence (Anne.Cadoret@univ-amu.fr)

${ }^{\mathrm{b}}$ UMR 7533 LADYSS CNRS-Universités Paris 1, Paris 7, Paris 10 et Paris 8, 2 rue de la Liberté - 93200 SAINT-

DENIS (segolene.darly@univ-paris8.fr)

'IRISSO UMR 7170 CNRS-Université Paris-Dauphine, Place du Maréchal-de-Lattre-de-Tassigny, 75775 Paris cedex16(thierry.kirat@dauphine.fr)

dART-Dev UMR 5281 CNRS-Université Montpellier 3, Route de Mende, 34199 MontPellier cedex 5 (lavaud. letilleul@club-internet.fr)
}

Résumé : Les littoraux sont des espaces fortement anthropisés, en mutation rapide, soumis à de nombreux enjeux et intérêts. Encadrés par une réglementation spécifique, ils sont un terrain propice à l'émergence d'une diversité de conflits qui se révèlent au total plus nombreux que dans l'arrière-pays. Certains d'entre eux sont portés par les acteurs devant les tribunaux. En s'appuyant sur l'étude de l'activité contentieuse de deuxième degré dans le département de l'Hérault, l'article propose de donner un éclairage sur la spécificité des conflits d'usage littoraux par rapport aux conflits qui se déroulent à l'intérieur des terres en tenant compte des dynamiques socio-économiques de ces espaces.

\begin{abstract}
Coastal zones undergo rapid changes due to great human pressure. Ruled by specific land use regulations, they are the scene of a variety of conflicts which tend to be more numerous than those located in the further inland. Some of them are brought to court by actors. Among the literature dedicated to opposition process, little has been written on the interactions between the socioeconomic dynamics of further inlands and coastal zones. This study demonstrate the specificity of littoral conflicts compared to further inland conflicts, capitalizing the study of administrative and civil courts activity in the French administrative department of Hérault.
\end{abstract}

Mots clés : contentieux - conflits d'usage - dynamiques territoriales - littoral - Hérault

Keywords: litigation - land use conflicts - territorial dynamics - littoral - Hérault 


\section{INTRODUCTION}

En tant qu'espaces d'interface en mutation rapide depuis les années 1960, soumis à une très forte pression anthropique, les littoraux sont sujets à une pluralité d'usages et à une diversité de dispositifs de gestion, particulièrement propices à l'émergence de conflits d'usage. Leur diversité donne un indice de la multiplicité des intérêts en présence sur les espaces côtiers. L'accès et l'exploitation des ressources par des usagers traditionnels tels que les chasseurs ou les pêcheurs; la périurbanisation et l'artificialisation des surfaces agricoles et des espaces naturels; les implantations d'équipements collectifs (infrastructures hôtelières, l'implantation de centres de traitement des déchets ou de fermes éoliennes, etc.) sont autant d'enjeux pour l'affectation et l'usage de l'espace littoral (Vallega, 1999; Goldberg, 1994; Paoli et al., 2008; Cadoret et al., 2008; Cadoret 2011; Lavaud-Letilleul et Cadoret, 2011).

Les conflits d'usage, dont l'intérêt principal est de donner une indication sur les enjeux socio-spatiaux et les stratégies d'acteurs sur un espace donné, font l'objet de travaux qui se sont développés de façon relativement indépendante (dont Ley et Mercer, 1980 ; Villeneuve et Côté, 1994; Caron et Torre, 2002; Trudelle, 2003; Cadoret, 2006; Kirat et Torre, 2006 ; Kirat et Torre, 2007 ; Darly, 2008; Villeneuve et al., 2009). Ces travaux se répartissent le plus souvent en fonction du degré d'urbanisation de l'espace concerné. Et de ce point de vue, leurs conclusions sont utiles pour l'étude des littoraux qui comportent aussi bien des espaces ruraux qu'urbains et périurbains. Dans les villes, l'étude des dynamiques conflictuelles aide à la compréhension des transformations urbaines (Villeneuve et Coté, 1994). Elle offre des éclairages sur les réussites et les échecs de projets urbanistiques en révélant des dysfonctionnements de l'action publique (Renaud, 2001) et fournit des éléments de compréhension sur les dynamiques participatives, les pratiques des acteurs au sein d'un quartier et sur les interactions sociales (Sénécal, 2002) permettant d'étudier la production de nouvelles territorialités (Mélé, 2004) ou d'observer les liens entre les antagonismes (Trudelle, 2003). Les espaces périurbains reçoivent quant à eux une attention particulière du fait qu'ils sont devenus des lieux emblématiques du développement de la ville diffuse depuis la fin des années 1970 (Kayser, 1989;
Nikolaïdou, 2009). Ce sont des lieux de tensions croissantes entre usagers concurrents, attirés par les disponibilités foncières de territoires excentrés mais bien connectés par les réseaux à l'agglomération (Cadène, 1990; Donadieu et Fleury, 2003; Darly, 2009). A ces tensions s'ajoutent des problèmes aigus de localisation des infrastructures métropolitaines, privées ou publiques, lorsque la gentrification des populations résidentielles périurbaines s'accompagne d'un fort abaissement des seuils de tolérance aux nuisances environnementales (Pham et Kirat, 2008). Dans les espaces ruraux enfin, l'articulation complexe entre les fonctions agricoles, résidentielles et de protection des espaces occasionne des conflits, et ce particulièrement dans les zones touristiques et dans les zones où se développent de façon accrue des usages récréatifs (Le Caro, 2002; Caron et Torre, 2002).

Espaces en mutation rapide, les littoraux concentrent donc des enjeux propres aux espaces urbains, périurbains et ruraux dont les dynamiques territoriales contribuent à l'émergence de conflits d'usage. Se pose alors la question de savoir si l'interface terre-mer donne naissance à des enjeux spécifiques aux littoraux. La proximité de la mer contribue-telle à rendre singulier les conflits qui s'y déroulent ? Bruno Charlier (1999) a démontré que les conflits environnementaux y sont plus fréquents, mais leurs caractéristiques sont-elles différentes (mode d'engagement, acteurs impliqués, objet des conflits, etc.) ? Force est de constater qu'à notre connaissance, peu de travaux s'attachent à mettre en comparaison les dynamiques antagoniques du littoral avec celles de l'arrière-pays. Cet article propose de pallier ce manque en donnant un premier éclairage sur la spécificité des conflits d'usage littoraux par rapport aux antagonismes se déroulant à l'intérieur des terres. Les travaux sur les antagonismes portent plus généralement sur un type de conflit que sur un type d'espace, et plus rarement sur un vaste territoire (Cadoret, 2011). Or l'analyse d'un ensemble de conflits sur un vaste territoire offre notamment la possibilité d'établir des comparaisons spatiales en son sein, d'où l'intérêt d'une étude à l'échelle d'un département littoral.

Cette analyse porte sur l'un des 26 départements littoraux de métropole : le département de l'Hérault (figure 1). Pour analyser la conflictualité dans le département de l'Hérault, nous avons observé l'acti- 
vité des tribunaux de deuxième instance, c'est-à-dire les actions en justice les plus longues et tendues entre les parties. La méthode et l'approche de la géographie de l'action contentieuse sont exposées dans la première section de l'article. La deuxième aborde les résultats obtenus à l'échelle du département de l'Hérault. À partir de ces données empiriques, la dernière partie permet de distinguer les caractéristiques des « conflits littoraux » au regard de ceux de l'arrière-pays, afin de mieux comprendre la relation entre configurations socio-spatiales d'un côté et logiques d'action et d'acteurs de l'autre.

\section{Cadrage conceptuel et métho- DOLOGIQUE : LITTORAL, CONFLITS D'USAGE ET CONTENTIEUX}

\section{Un terrain : le littoral héraultais}

L'analyse porte sur le département de l'Hérault qui constitue le centre de gravité démographique de la région Languedoc-Roussillon. Avec une population de 1 million d'habitants en 2009 sur près de 2,6 millions pour la région, ce département connaît la troisième plus forte croissance démographique de l'hexagone avec un taux de croissance de 1,6\% par an pour la période 1999-2006 (INSEE, 2009). Il s'explique essentiellement par un apport migratoire exceptionnel. Entre 1990 et 2005, l'excédent migratoire annuel s'élève à plus de 11000 personnes dans le département de l'Hérault, contre 25000 pour la région. Or, cette pression s'exerce en particulier sur la frange littorale qui concentre les densités de population les plus importantes, par rapport aux zones de garrigues et de montagne des hauts cantons de l'arrière-pays.

Deux facteurs concomitants l'expliquent : l'attraction exercée par le bord de mer et l'extension périphérique du Grand Montpellier. Ces dynamiques territoriales contribuent à la transformation des usages du sol et à une multifonctionnalité qui, directement ou indirectement, modifient certaines pratiques, représentations et/ou systèmes écologiques (recul des surfaces agricoles exploitées, augmentation du prix du foncier, impacts paysagers, pollutions, modification des écosystèmes, dégradation de zones humides, perturbation de pratiques traditionnelles, etc.).
Cette pression démographique soutenue constitue de plus une rupture complète par rapport aux dynamiques globales d'occupation de l'espace régional. La région languedocienne est traditionnellement tournée vers l'intérieur, comme le montrent la répartition de l'activité viticole et l'implantation des principales villes datant de l'époque médiévale (Le Roy Ladurie, 1962; Rouzier, 2002). Il a fallu attendre les années 1960 pour que le plan d'aménagement touristique de portée nationale piloté par la Mission Racine investisse ce littoral à lido en artificialisant et peuplant cette côte lagunaire (Racine, 1980). Ce basculement récent du centre de gravité de l'intérieur des terres vers la zone côtière apparaît comme un facteur supplémentaire de tensions sur le plan socio-culturel, entre des usages traditionnels de l'espace littoral (chasse, petite pêche côtière...) et des pratiques beaucoup plus récentes (tourisme de masse, urbanisation...).

Les évolutions observées sur le département de l'Hérault durant les 50 dernières années constituent autant d'enjeux en termes d'aménagement, de développement économique et de protection des espaces qui motivent les acteurs à s'engager dans des actions contentieuses afin de défendre des intérêts qui s'opposent.

\section{Une approche : la géographie de l'action contentieuse de seconde instance}

L'analyse des contentieux suscite l'intérêt de chercheurs de disciplines variées, comme le droit et les sciences politiques (Leost, 1998), l'économie (Kirat et Lefranc, 2004), la sociologie (Lascoumes et Timbard, 1993; Melot et Pelisse, 2008) ou la géographie. Dans le domaine de l'étude des conflits d'usage, les géographes restent peu nombreux à travailler sur ce matériau juridique, il est toujours associé à d'autres sources d'information, comme les entretiens ou la presse quotidienne régionale (Rialland, 2003; Cadoret, 2006; Darly, 2009; Torre et al., 2010). La géographie des conflits à partir de sources juridiques, et en particulier la géographie de l'action contentieuse, reste à ce jour un terrain de recherche encore largement à explorer. La principale difficulté est que ce corpus suppose en pratique un travail interdisciplinaire associant des spécialistes des questions juridiques et des questions socio-spatiales. C'est ce que nous nous sommes proposés de faire dans le cadre de cet article, en associant un économiste du droit et des géographes. 
L'étude des actions portées devant les tribunaux offre de précieuses informations pour évaluer la conflictualité sur un espace. Elle permet notamment de repérer à la fois les objets d'oppositions et les acteurs impliqués, ainsi que leur registre d'argumentation. Elle permet d'étudier le conflit en tant que processus. Elle offre un regard à la fois sur un mode d'émergence des conflits (l'objet des requêtes), une forme d'opposition (l'action en justice étant un mode d'expression des conflits parmi d'autres) et une modalité de régulation des conflits (l'arbitrage du juge) (Cadoret, 2011). La spatialisation des contentieux permet quant à elle de localiser ces actions et de repérer d'éventuelles régularités et/ ou disparités géographiques.

Sur le plan du corpus de données offert, travailler sur les conflits d'usage à partir des actions contentieuses comporte certes quelques limites: les actions en justice, qui plus est menées dans les juridictions de seconde instance, ne donnent qu'un aperçu des conflits d'usage existant sur un territoire donné. Mais l'analyse des actions contentieuses de seconde instance présente en revanche un avantage majeur pour un travail de différenciation régionale centré sur le binôme littoral/arrière-pays tel que le nôtre : elle permet de repérer certaines caractéristiques des dynamiques conflictuelles à l'échelle d'un vaste territoire. Il serait certes scientifiquement pertinent d'apprécier les conflits d'usage à partir d'autres sources d'informations (presse, entretiens...). Mais en termes de faisabilité, l'étude des actions portées devant les tribunaux permet de dépasser la difficulté d'un travail fastidieux de recueil de données pour un ensemble de conflits, en offrant les premières pistes de compréhension des conflits à l'échelle d'un vaste ensemble territorial.

\section{Sources et données : le contentieux administratif et judiciaire de seconde instance}

Les sources utilisées pour analyser les conflits d'usage dans le département de l'Hérault sont de nature juridique. Elles portent plus précisément sur le contentieux administratif et judiciaire relatif aux conflits localisés dans une ou plusieurs communes du département au cours de la période 1981-2005

1. La base de données exploitée contient toutes les décisions des juridictions de second degré rendues depuis 1981. Les conditions d'abonnement à la qui ont donné lieu à une action devant les juridictions judiciaires ou administratives de seconde instance. Les décisions ont été recueillies en texte intégral sur la base LamylineReflex, qui comprend l'intégralité des arrêts rendus par le Conseil d'Etat et les Cours administratives d'appel depuis leur création en 1989, et la Cour de cassation. Notons qu'une limite de la source LamylineReflex est de ne pas comprendre les décisions des juridictions de première instance dans les ordres judiciaires (Tribunaux d'instance et de grande instance) et administratifs (tribunaux administratifs). Il est important de noter que l'activité des tribunaux de deuxième instance concentre notre analyse sur les actions en justice les plus longues et tendues entre les parties. Le corpus ainsi constitué comprend au total 125 décisions. Leur distribution est détaillée dans le tableau 1.

Deux données sont particulièrement importantes : l'objet du contentieux et le domaine juridique des requêtes. L'objet du contentieux porte sur la dimension matérielle ou factuelle du contentieux. Les variables qui s'y rapportent concernent les enjeux réels du conflit, qui ont été distingués des enjeux juridiques. Ces derniers relèvent de ce que nous appelons le domaine de la requête, qui saisit la mise en forme, dans le langage du droit, des demandes adressées aux juridictions. Par exemple, un projet de création d'une installation classée pour la protection de l'environnement peut être contesté au moyen d'une argumentation juridique centrée sur les règles d'urbanisme, alors que l'enjeu porté par une association de protection de l'environnement est la préservation de l'environnement naturel. Dans un tel cas, l'objet du contentieux est la préservation de la nature, alors que les moyens de la requête relèvent du droit de l'urbanisme.

Les données issues du contentieux de seconde instance ont été complétées par des données de contexte au niveau communal, qui sont présentées en détail dans le tableau en annexe. Collectées auprès de l'Institut Français pour l'Environnement (Corine Land Cover), du Ministère en charge de l'environnement (MEDDTL), de la Direction Générale des Impôts (DGI) et de l'Institut National de la Statistique et des Etudes Economiques (INSEE), elles sont relatives à la démographie, aux modes d'occupation des sols, à l'appartenance ou

base ne permettaient pas, dans le cadre de cette étude, d'avoir accès aux décisions rendues après 2005. 


\begin{tabular}{|c|c|c|}
\hline Contentieux de l'Hérault (1981-2005) & Juridiction de deuxième instance & Nombre d'arrêts \\
\hline Contentieux administratif & & 80 \\
\hline \multirow{3}{*}{ dont } & Cours administrative d'appel & 39 \\
\cline { 2 - 3 } & Marseille* $^{*}$ & 30 \\
\cline { 2 - 3 } & Bordeaux $^{*}$ & 9 \\
\cline { 2 - 3 } & Conseil d'Etat & 41 \\
\hline Contentieux judiciaire & & 45 \\
\hline \multirow{2}{*}{ dont } & Civil & 43 \\
\hline Total & Pénal $^{* *}$ & 2 \\
\hline
\end{tabular}

Tableau 1 : Distribution des décisions de justice de deuxième degré dans le département de l'Hérault (1981-2005)

( ${ }^{*}$ Le recours contre les jugements du TA de Montpellier étaient du ressort de la Cour administrative d'appel de Bordeaux jusqu’à la création de la Cour administrative d'appel de Montpellier. ${ }^{* *}$ Les deux affaires pénales sont des arrêts de la chambre criminelle de la Cour de cassation relatifs à des infractions à la législation sur l'environnement. Il s'agit d'agissements de responsables d'exploitation de services publics délégués qui ont conduit à des pollutions de cours d'eau)

Distribution of appeal and supreme courts on cases located in Hérault (1981-2005)

non à une aire urbaine, aux revenus des ménages et aux autorisations de construction. Chaque commune ayant connu au moins un conflit porté devant les tribunaux de seconde instance a été identifiée comme littorale ou intérieure. Les communes littorales sont celles qui sont définies comme telles par la loi Littoral de $1986^{2}$. Elles sont au nombre de 25 dans le département de l'Hérault ${ }^{3}$.

\section{LA GÉOGRAPHIE DE L'ACTION CONTENTIEUSE DE SECONDE INSTANCE DANS LE DÉPARTEMENT DE L'HÉRAuLT}

\section{Une concentration dans le « monde plein » du département}

Les concentrations de contentieux de seconde instance sont localisées dans le «monde plein » du département de l'Hérault (figure 1), à savoir :

- dans les principales agglomérations (Montpellier et Béziers);

2. Selon les termes de la loi n 86-2 du 3 janvier 1986 relative à l'aménagement, la protection et la mise en valeur du littoral (article 2), les communes littorales, au sens de cette loi, sont les communes de métropole et des départements d'outre-mer : riveraines des mers et océans, des étangs salés, des plans d'eau intérieurs d'une superficie supérieure à 1000 hectares, riveraines des estuaires et des deltas lorsqu'elles sont situées en aval de la limite de salure des eaux et participent aux équilibres économiques et écologiques littoraux. La liste de ces communes est fixée par décret en Conseil d'État, après consultation des Conseils municipaux intéressés.

3. Du nord au sud : Lansargues, Marsillargues, Candillargues, La Grande Motte, Mauguio, Palavas-les-Flots, Pérols, Lattes, Villeneuve-lès-Maguelonne, Vic-la-Gardiole, Mireval, Frontignan, Sète, Balaruc-le-Vieux, Balaruc-les-Bains, Loupian, Bouzigues, Mèze, Marseillan, Agde, Vias, Portiragnes, Sérignan, Valras-Plage, Vendres).
- et au niveau du littoral héraultais (avec des points de concentration du nord au sud, à MauguioCarnon, Sète et Agde).

L'arrière-pays où se trouvent les densités les plus faibles est peu sujet au contentieux de seconde instance, à l'exception de la vallée de l'Hérault, où se cale le tracé de l'A75, qui tend à devenir, du fait de sa bonne accessibilité, une zone d'extension du périmètre urbanisé de l'agglomération de Montpellier.

Tous types de contentieux confondus, il apparaît de façon assez logique que le nombre des contentieux est corrélé à l'importance des densités au sein du département (figure 1). Ces zones de densité sont constituées par les quatre grands pôles urbains du département (Montpellier, Béziers, Sète et Lunel) et leurs couronnes périurbaines (voir figure 1 et figure 2 - planche I), ainsi que par les communes situées dans l'aire d'influence des grandes voies de communication (A75 et A750, prolongement de l'autoroute A75 reliant l'agglomération de Montpellier au Haut-Languedoc). Un chapelet de villes et d'aires urbaines, où la densité est supérieure à la moyenne nationale, se répartit selon un axe parallèle à celui du littoral. Cette concentration de la population contraste avec l'ensemble intérieur du département, caractérisé par un large étalement des densités de population, selon un gradient classique urbain-périurbain-rural.

Cette correspondance entre activité contentieuse et densité de population permet de mettre en évidence une première distinction entre intérieur et littoral : alors que l'ensemble intérieur est carac- 


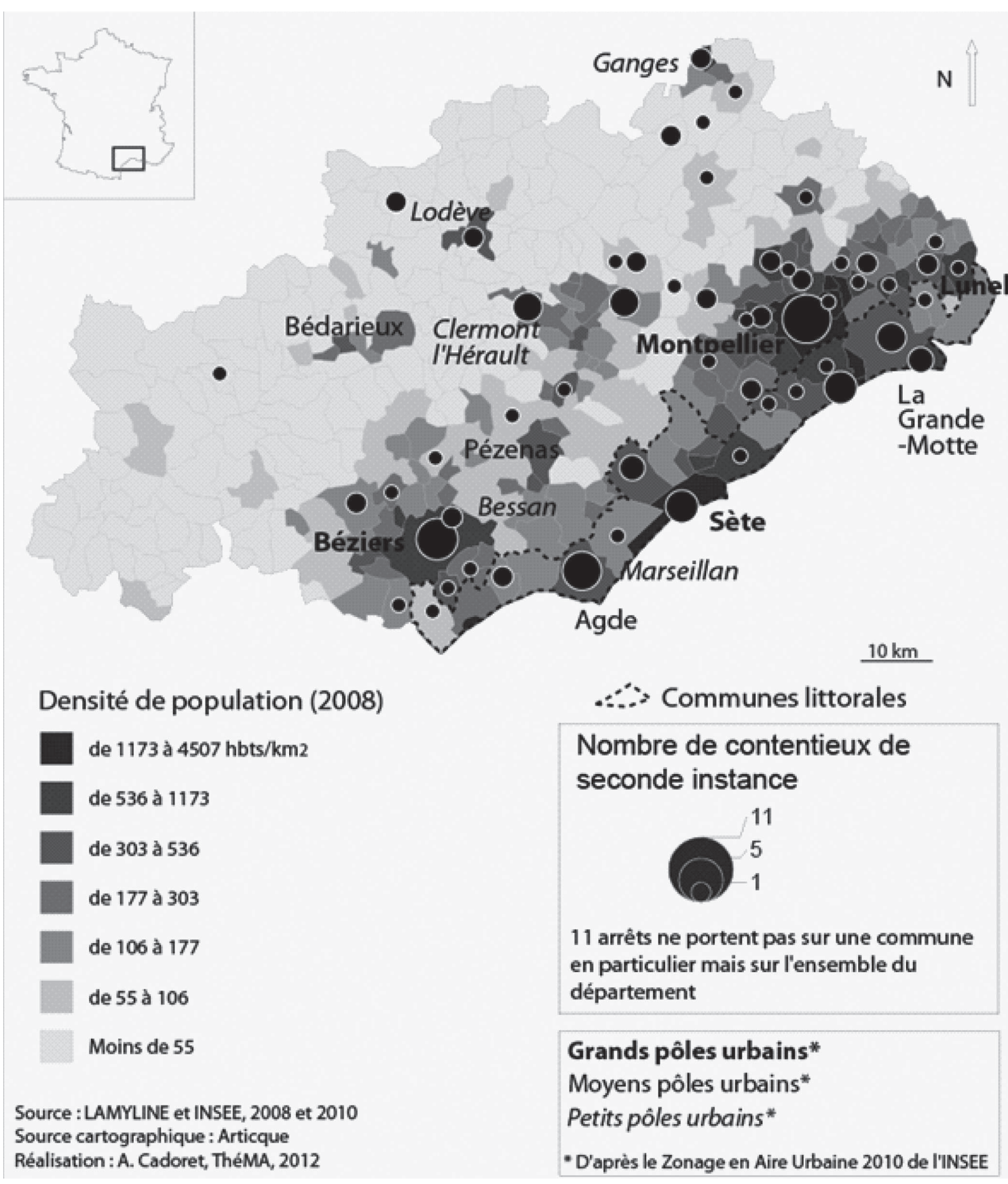

Figure 1 : Localisation du contentieux de seconde instance (1981-2005) et densités de population en 2008 concernant les communes du département de l'Hérault

Appeal litigation map (1981-2005) with population density for the municipalities (2008) of the French department of Hérault 
térisé par une moins grande activité des tribunaux de seconde instance concernant les conflits d'usage et par une alternance entre zones plus ou moins conflictuelles (à relier au gradient de densité de population des communes), la zone littorale est plus fortement et plus uniformément conflictuelle.

Si l'on se penche ensuite sur la répartition des différentes catégories de contentieux (ici distinguées en fonction du domaine de leur requête), de nouvelles logiques de localisation apparaissent (figure 2 - planche I). Les contentieux liés à l'urbanisme sont localisés en milieu urbain et sur le front d'urbanisation, c'est-à-dire aux marges des plus grandes agglomérations, dans la zone d'influence des grands pôles urbains de Montpellier et de Béziers notamment, qui s'étend sur les communes rurales et les petites unités urbaines de l'arrière-pays (figure 2), ainsi que sur le littoral qui représente la zone où s'exerce aujourd'hui la plus forte pression foncière. Les contentieux (judiciaires) liés aux servitudes de passage et au voisinage se situent en milieu urbain (Montpellier) et dans les zones périphériques pour l'accès à des parcelles agricoles ou des zones naturelles. Enfin, les contentieux liés aux Installations Classées (ICPE) et à la dépollution de site en activité répondent quant à eux à une logique de répartition plus ponctuelle, en fonction de la localisation des établissements ou des projets d'établissement concernés.

Si l'on se focalise sur la nature du contentieux dans les communes de forte densité, la cartographie révèle une distinction entre les conflits des communes du littoral et de l'arrière-pays. Dans l'arrière-pays, le contentieux de deuxième instance est plus diversifié en termes de domaines de requête, les conflits où l'urbanisme est mobilisé n'y étant que minoritaires par rapport aux contentieux liés aux servitudes et à la dépollution. À l'inverse, le contentieux de seconde instance des communes littorales reste en majorité un contentieux qui utilise le langage de l'urbanisme (à l'exception du cas de MauguioCarnon où, sur quatre conflits juridiques, un seul concerne la révision du plan d'occupation des sols, contestée par le préfet, les autres conflits étant relatifs à des servitudes de passage). Ce constat nous pousse à émettre l'hypothèse d'une géographie spécifique des conflits d'urbanisme selon que l'on considère le littoral et l'arrière-pays, hypothèse qui fait l'objet de la section suivante.

\section{Le contentieux d'urbanisme, l'exception littorale}

L'observation de la répartition par commune de la part du contentieux de seconde instance où le Code de l'Urbanisme est mobilisé (figurés rouge sur la figure 2 - planche I) montre que le littoral constitue une exception au sein du département de l'Hérault. En effet, la figure 2 met en évidence une concentration des contentieux de seconde instance mobilisant le droit de l'urbanisme sur les communes littorales. La moitié de ces affaires est située sur la zone côtière.

À l'échelle du département de l'Hérault, il apparaît clairement que le contentieux de seconde instance relevant du Code de l'urbanisme est inversement proportionnel au degré d'urbanisation de la commune. Dans des communes urbaines de l'agglomération de Montpellier, cette part s'avère très limitée, par rapport à ce que l'on observe dans des communes plus rurales de l'arrière-pays où les contentieux liés à l'urbanisme représentent la majorité des affaires ${ }^{4}$. Ce résultat s'explique par deux facteurs :

- les communes moins urbanisées de l'arrière-pays sont aujourd'hui celles qui sont le plus soumises à la pression urbaine et à la mutation des espaces ouverts pour la construction de nouveaux logements et d'infrastructures, les actes administratifs d'urbanisme y sont donc plus nombreux.

- les communes très urbanisées (comme celles de l'agglomération de Montpellier) sont de facto les plus peuplées, les plus denses et celles où l'artificialisation des sols est la plus importante. Les possibilités de développement urbain y sont moindres. Cependant, la proportion de conflits de voisinage et de servitudes liés à la forte proximité géographique entre individus y est plus importante.

En revanche, dans les communes littorales de l'Hérault, la situation est toute autre. Dans un contexte de pression foncière extrême, la part du contentieux liée à l'urbanisme est surreprésentée quel que soit le type de commune, y compris dans les communes urbanisées des pôles urbains.

\footnotetext{
4. Les conflits récurrents dans l'agglomération de Montpellier portent sur la dégradation de l'accessibilité des maisons du fait d'aménagements routiers (CAA Marseille, 10 mai 2000), la pollution due à une entreprise industrielle (CAA Marseille, 15 mars 1996), des nuisances de voisinage diverses (Cour de cassation, divers arrêts) et quelques cas d'intolérance à de création de centres d'accueil de travailleurs migrants (Conseil d'Etat, 11 juillet 1979) ou d'aide aux toxicomanes (Cassation, SOS Drogues International, 16 janvier 2001).
} 
Néanmoins, et ce constat peut paraître contre-intuitif, il faut souligner que les dispositions de la « loi Littoral », codifiées dans le Code de l'urbanisme", sont très peu activées dans le contentieux de l'urbanisme : les dispositions de la « loi Littoral » ne sont présentes que dans 4 des 21 décisions qui évoquent, au titre de normes juridiques principales, les règles générales d'aménagement et d'urbanisme.

Cette analyse géographique de l'action contentieuse de seconde instance dans le département de l'Hérault et celle concernant la localisation des requêtes traitées par les tribunaux de second degré révèlent plusieurs résultats. Au niveau quantitatif, le nombre de contentieux de seconde instance varie proportionnellement avec la densité des communes. Ils se concentrent donc dans les zones les plus densément peuplées dont fait partie le littoral, au même titre que les agglomérations de l'arrière-pays. En revanche, au niveau qualitatif, le contentieux de seconde instance présente des caractéristiques différenciées selon que l'on se situe sur le littoral ou dans l'arrière-pays. Dans l'arrière-pays, la nature des contentieux varie avec le niveau d'urbanisation de la commune : les contentieux des communes urbaines sont diversifiés (et mobilisent minoritairement l'urbanisme comme argument juridique), alors que les conflits des communes rurales sont essentiellement liés à l'urbanisme et aux installations classées. Dans l'espace littoral, les contentieux des communes urbaines utilisent en revanche majoritairement le droit de l'urbanisme. Dans ce deuxième cas, la géographie spécifique du contentieux de seconde instance sur le littoral ne saurait s'expliquer uniquement par la dynamique d'urbanisation de la côte, mais serait plutôt liée à la spécificité des conflits qui s'y déroulent. L'analyse de cette spécificité fait l'objet de la section suivante.

\section{Conflits hitToraux ET CONFLITS INTÉRIEURS}

La géographie de l'action contentieuse de seconde instance à l'échelle du département de l'Hérault pose la question de l'influence du contexte littoral sur la répartition des conflits. La pression urbaine qui s’y manifeste se traduit-elle par une surreprésen-

5. Code de l'urbanisme : Chapitre VI : Dispositions particulières au littoral

- Titre IV : Dispositions particulières à certaines parties du territoire du

Livre I Règles générales d'aménagement et d'urbanisme. tation du contentieux de deuxième degré? La cohabitation entre usages traditionnels (chasse, pêche, agriculture...) et récents (tourisme, urbanisation...) est-elle source d'antagonismes? La réglementation spécifique encadrant le développement des communes littorales est-elle porteuse de différends? Pour cela, nous avons cherché à mettre en évidence les caractéristiques spécifiques des conflits localisés dans des communes littorales par rapport à celles des conflits localisés dans les communes de l'arrièrepays ${ }^{6}$. Par souci de simplification, nous nommerons ces deux catégories de conflits " conflits littoraux » et « conflits intérieurs».

\section{Les « conflits littoraux » sont-ils spécifiques?}

Au niveau quantitatif, la façade maritime du département de l'Hérault est tout d'abord globalement plus touchée que l'arrière-pays par le contentieux de seconde instance. Il ressort en effet de notre étude que près de $16 \%$ des communes du département sont concernées par au moins un conflit porté devant les tribunaux de seconde instance (soit 55 communes sur 343). Et, alors que seulement $13 \%$ des communes non littorales de l'Hérault sont concernés par au moins un contentieux, c'est le cas de $60 \%$ des communes littorales. Les communes littorales représentent $7 \%$ des communes du département, mais concentrent un tiers des contentieux localisables ${ }^{7}$ de l'Hérault. Le littoral forme donc de façon remarquable le théâtre privilégié du contentieux de seconde instance au niveau départemental.

Au niveau de l'objet des conflits, une analyse géographique sur l'ensemble des contentieux de seconde instance et des analyses factorielles menées sur le contentieux administratif révèlent par ailleurs l'existence d'une discontinuité entre les conflits situés sur le littoral et les conflits qui surviennent dans l'arrière-pays du département.

L'analyse géographique des contentieux de seconde instance a révélé précédemment que les contentieux de seconde instance mobilisant le droit de l'urbanisme caractérisent les communes litto-

6. Dans le département de l'Hérault, il y a 318 communes dans l'arrière-pays et 25 communes littorales.

7. Onze contentieux de seconde instance ne portent pas sur une commune en particulier, mais sur l'ensemble du département. Il s'agit de contentieux portant sur les dates d'ouverture de la chasse. 
rales. Une autre spécificité apparaît à la lecture de la figure 3 (planche II). Cette carte porte uniquement sur les affaires de seconde instance où le droit de l'urbanisme est mobilisé (soit 54 affaires sur 125) et précise l'objet des contentieux.

Il s'avère que les objets des contentieux relevant du Code de l'urbanisme ne concernent pas toujours des opérations ou document d'urbanisme. Que se soit dans l'arrière-pays ou sur le littoral, seulement près de $40 \%$ des contentieux où le droit de l'urbanisme est mobilisé concernent des conflits dont l'objet est l'urbanisme (respectivement $40 \%$ et $36 \%$ ). Les contentieux où l'urbanisme est pris sous l'angle de la mise en œuvre juridique ou celui des objets matériels concernent plus particulièrement les communes situées dans les grandes aires urbaines. Une spécificité littorale apparaît lorsque l'on s'intéresse aux objets des conflits pour lesquels les arguments juridiques de l'urbanisme sont utilisés.

En effet, alors que dans les communes intérieures, les arguments juridiques portant sur l'urbanisme sont mobilisés pour une diversité de litiges, sur le littoral, le droit de l'urbanisme est pour moitié utilisé dans des contentieux dont l'objet est la gestion et la préservation du milieu naturel. Il s'agit par exemple de la création de la réserve naturelle du Bagnas, de la réserve de Roque-Haute, des espaces sensibles menacés par la construction de la Zone d'Aménagement Concertée (ZAC) du triangle du Villeroy à Sète ou d'un lotissement à Palavas. Dans l'arrière pays, seulement $15 \%$ des contentieux de seconde instance mobilisant le droit de l'urbanisme ont pour objet la préservation du milieu. Les arguments juridiques relevant de l'urbanisme sont mobilisés pour des affaires portant sur des nuisances liées par exemple à l'implantation d'un centre de gestion des déchets (Lunel-Viel), à la construction d'un centre autoroutier (Clermont l'Hérault), à l'implantation d'un foyer de migrantes (Montpellier).

Cette analyse sur l'ensemble des contentieux de seconde instance complète celle réalisée sur le contentieux administratif par les analyses factorielles. D'un point de vue quantitatif, l'observation des données du contentieux administratif révèle que l'urbanisme, qu'il soit pris sous l'angle de la mise en œuvre juridique ou sous celui des objets matériels, apparaît comme le premier domaine conflictuel dans le département de l'Hérault. Les objets de conflits portant sur l'urbanisme représentent un quart des affaires prises sous un angle matériel ou factuel. Quant à l'argumentation de la saisine des juridictions qui utilise le langage du droit de l'urbanisme, elle représente plus de la moitié des affaires prises sous l'angle du domaine des requêtes. C'est là un indice probant du caractère générique et transversal du droit de l'urbanisme, qui dépasse les seules questions d'urbanisation, mais touche aussi, en termes fonctionnels, d'autres domaines tels que la protection de l'environnement et les installations industrielles classées.

Une analyse de correspondance a été réalisée afin de rendre compte de co-occurrences entre variables capturant les caractéristiques des contentieux administratifs de seconde instance - leur objet, le domaine juridique évoqué dans les requêtes, variables représentées par un carré blanc - et la localisation des communes sur le littoral ou dans l'arrière-pays. Le résultat obtenu (figure 4) révèle à la fois une nette corrélation entre les conflits pour la préservation de l'environnement (variable « objet de préservation » = Oui) et la localisation littorale des communes où ils se situent (variable «Littoral »= Oui), mais aussi une corrélation entre les conflits liés à l'activité industrielle et la localisation non- littorale des communes concernées (variable « Littoral » = Non).

Afin d'approfondir l'interprétation de ce résultat, les communes ont ensuite été caractérisées par un certain nombre de données qui permettent de dépasser la simple distinction littorale-non littorale et qui prennent la forme de variables continues (superficies protégées en hectare et en pourcentage de la superficie de la commune, nombre d'installations classées pour la protection de l'environnement ou relevant de la réglementation « Seveso ", solde naturel et solde migratoire, etc. $\left.{ }^{8}\right)$. Les corrélations entre variables actives ont ainsi démontré que les communes sièges d'au moins un conflit se répartissent en deux grandes catégories (figure 5). La première, qui regroupe une grande partie des communes non littorales, est caractérisée par un ensemble de variables situées à gauche et dans la partie inférieure du graphique : les communes y sont marquées par un fort dynamisme démographique (par un solde migratoire élevé), la présence d'installations classées pour la protection de l'environ-

8. La liste des variables figure dans une annexe à cet article. 


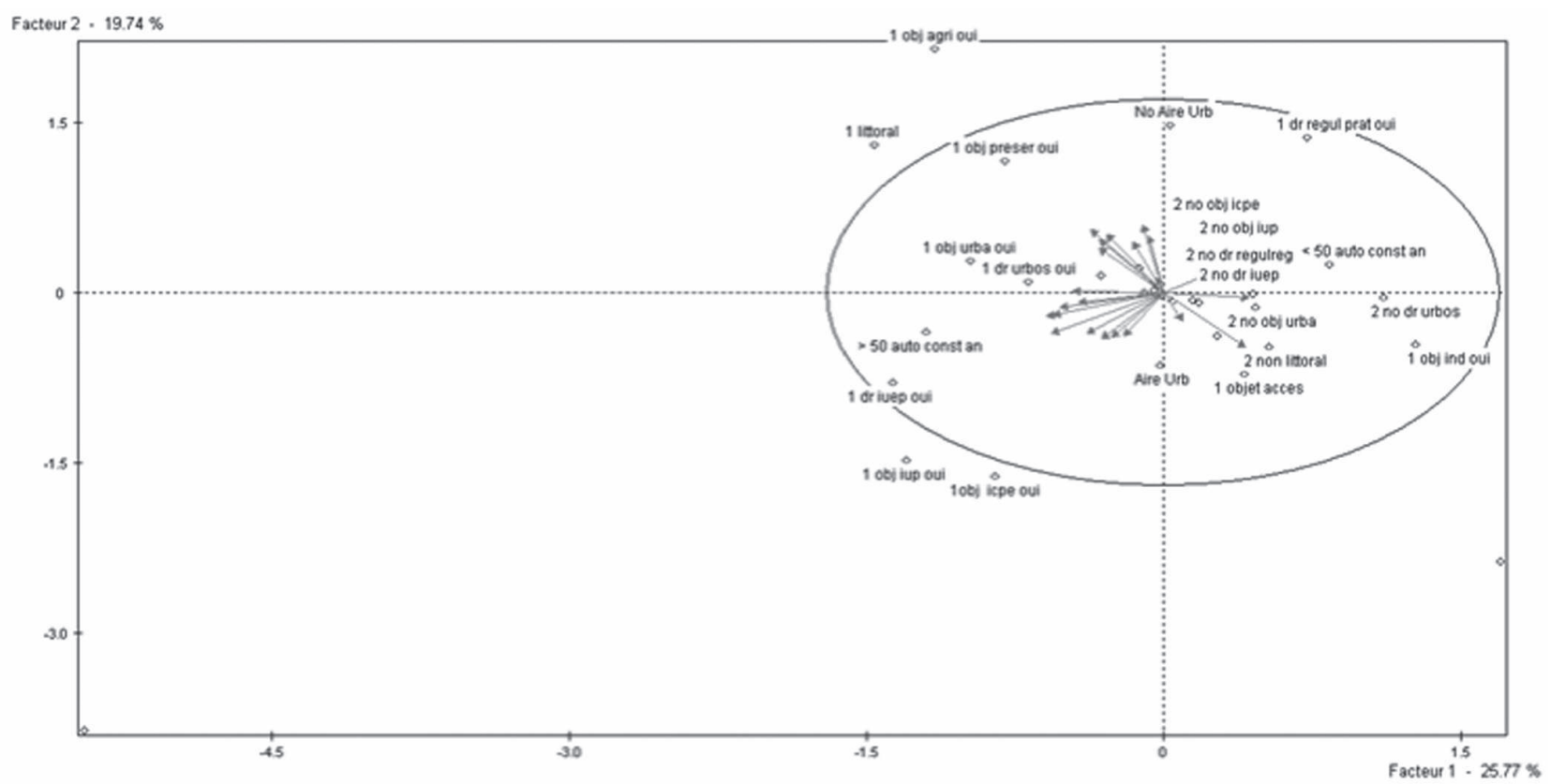

Figure 4 : Analyse de correspondances multiples Multiple correspondence analysis

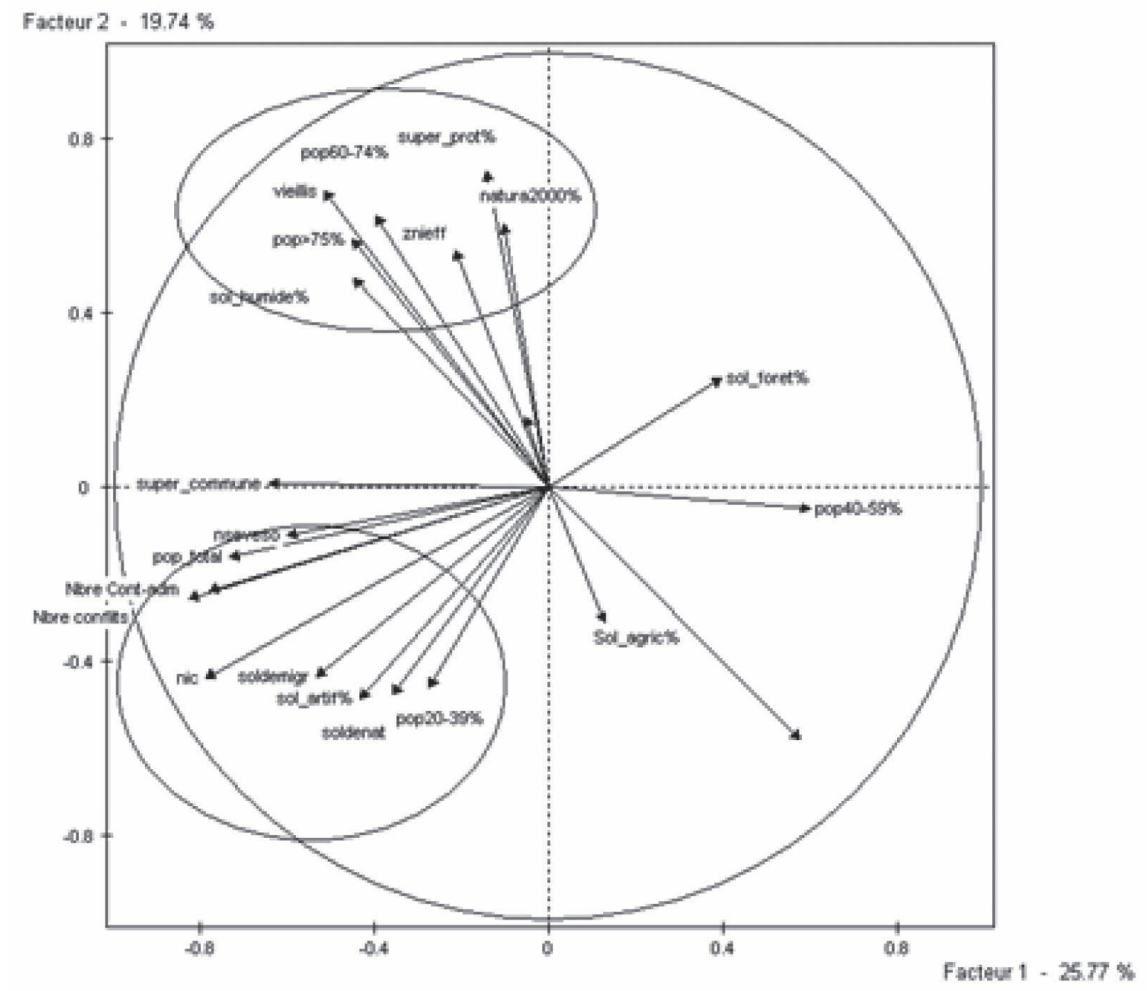

Figure 5 : Analyse en composantes principales : corrélations entre variables actives

Principal components analysis : correlation between active variables 
nement (y compris classées « Seveso »), et une artificialisation importante des sols. La seconde catégorie, qui regroupe essentiellement des communes littorales, est caractérisée par un autre ensemble de variables, positionné dans la partie supérieure du graphique et qui indiquent que les communes en question sont dotées d'espaces naturels sensibles et protégés, notamment des zones humides, et dont la population est plutôt âgée, mais pas forcément aisée économiquement.

La relation entre les caractéristiques des communes littorales et non littorales, que révèle la figure 5, et le type de conflits qui caractérisent ces deux catégories de communes (cf. figure 4), permet de préciser les éléments de distinction entre conflits littoraux et conflits intérieurs.

Les conflits intérieurs sont plus spécifiquement liés à des enjeux d'évitement des nuisances (infrastructures d'utilité publique, activités industrielles et extractives) dans des environnements urbains et périurbains, caractérisés par une population jeune qui se recompose rapidement sous l'effet de l'installation de nouveaux résidents (principalement sous la forme de maisons individuelles et de lotissements résidentiels). Les conflits littoraux se distinguent significativement de ces derniers par le fait qu'ils sont plus particulièrement liés à des enjeux de protection de l'environnement naturel, qui surviennent dans un contexte de population vieillissante, en faible augmentation et par ailleurs économiquement moins aisée que dans les communes intérieures.

À ce stade de l'analyse, il est difficile de donner une explication définitive à cette différence de profils entre conflits intérieurs et littoraux. On peut avancer comme explication que le rapport au territoire est plus identitaire pour les résidents âgés des littoraux que pour les résidents périurbains des communes intérieures, plus jeunes, et probablement engagés dans une activité professionnelle dans les grandes agglomérations comme Montpellier ou Béziers. Si les différences de méthodes empêchent une comparaison fiable entre l'Ile-de-France et l'Hérault ${ }^{9}$, une hypothèse peut être formulée, qui demandera à être vérifiée dans des travaux ulté-

9. Les travaux de Pham et Kirat (2008) sur l'Ile-de-France utilisent une méthode économétrique (ANOVA) pour tester l'influence des variables socioprofessionnelles sur l'occurrence des conflits et leur type, alors que le présent travail n’a pas utilisé une telle méthode. rieurs : les conflits d'environnement, mêlant droit de l'urbanisme et logique de protection, ne sont pas dans le département de l'Hérault conditionnés à la présence de catégories socioprofessionnelles intermédiaires ou supérieurs, alors que cela semblerait être le cas des conflits d'aménagement dans les communes intérieures.

\section{Les logiques d'acteurs et d'actions à l'œuvre : tensions entre développement économique, urbanisation et préservation de l'environnement}

Au-delà du constat opéré sur la nature des objets de conflits, il convient maintenant de s'intéresser aux acteurs de la conflictualité et aux logiques qui les portent. Nous analysons ici les acteurs engagés dans les actions en justice au regard des tensions qui traversent le département : entre développement économique et urbanisation d'un côté, préservation de l'environnement de l'autre.

Les contentieux engagent des acteurs publics et privés. Les acteurs publics sont les communes et leurs groupements, le préfet et l'administration centrale (les ministres concernés). Les acteurs privés sont des individus, des entreprises et des associations de défense d'intérêts locaux ou des associations de protection de l'environnement à portée non locale. L'identification des acteurs présents dans le contentieux administratif de seconde instance, que ce soit comme requérant ou comme défendeur, a été réalisée en distinguant deux catégories dans leur logique d'action. La première est une logique de développement économique et d'urbanisation, qui comprend les actes et décisions portant sur une action de développement par implantation d'activités ou d'infrastructures et des opérations d'urbanisme. La seconde catégorie concerne une logique de protection de l'environnement et du cadre de vie, qui comprend les actions de contestation, d'opposition, visant à conserver l'environnement ou les paysages qu'ils soient naturels ou anthropisés. Nous avons choisi de porter notre attention sur le seul contentieux administratif de seconde instance, parce que c'est devant les juridictions administratives que les confrontations de préférences et de logiques s'expriment, lors de la contestation d'actes administratifs et de décisions publiques. 


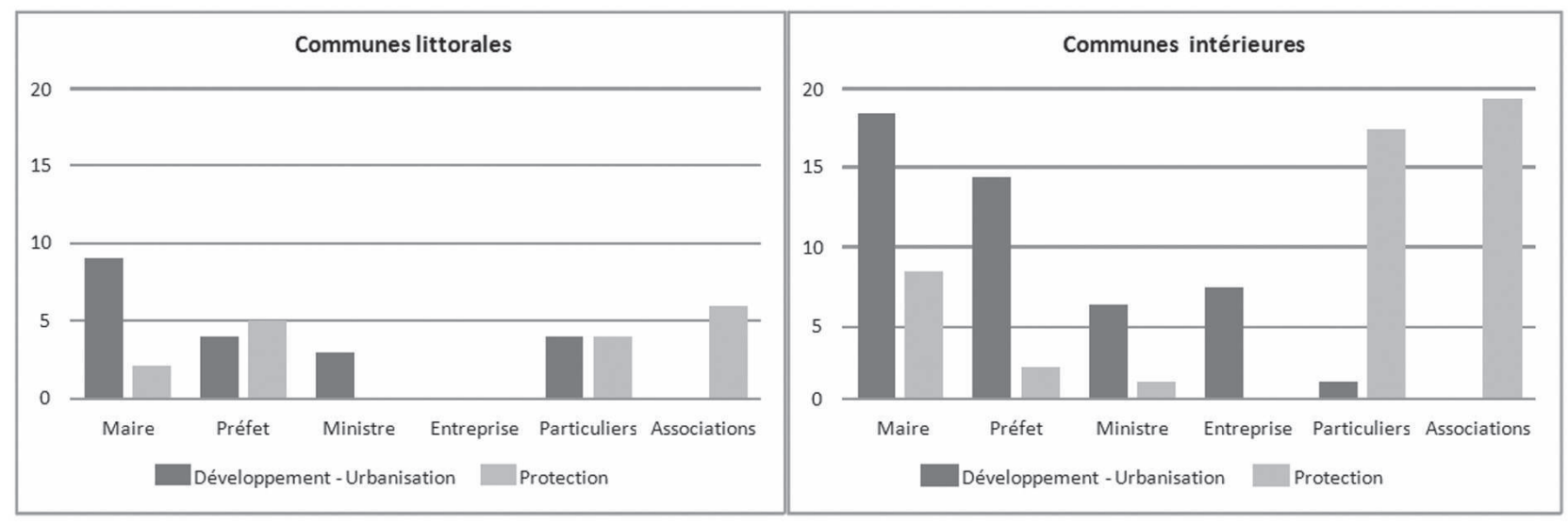

Figure 6 : Acteurs et logiques du contentieux administratif de deuxième instance dans le département de l'Hérault (1981-2005) Actors and main logics of the appeal litigation in the French department of Hérault (1981-2005)

La figure 6 met en évidence plusieurs éléments. Il n'est pas étonnant de constater que les associations de défense de l'environnement, en l'occurrence essentiellement locales et « circonstantielles », plutôt que nationales et généralistes (selon la typologie de Lascoumes, 1994), sont mus par des logiques de protection de l'environnement naturel et humain dans toutes les communes. En revanche, les particuliers qui dans l'arrière-pays ont une logique de protection et conservation, se répartissent sur le littoral à égalité entre volonté de développement et de protection. Du côté des acteurs publics, les maires qui sont partie prenante au contentieux, que ce soit comme demandeur ou comme défendeur, sont majoritairement « développementalistes » plutôt que « conservationnistes », surtout dans les communes littorales où le ratio " logique de développement et d'urbanisation/logique de protection » est de 4,5 contre 2,25 dans les communes de l'intérieur du département. Les logiques sont plus complexes au niveau de l'État. Pour les communes de l'arrièrepays, le préfet du département est quasiment systématiquement dans une logique de développement alors que sa position est plus équilibrée sur le littoral, les actions de conservation dépassant quelque peu les actions de développement. Les conflits qui mettent en jeu une action du ministre concerné, certes mineurs du point de vue quantitatif, sont plus équilibrés entre les deux logiques, aussi bien sur le littoral que dans l'arrière-pays. Certains acteurs ont donc une logique d'action identique, indépendante de leur localisation (les associations sont « protec- trices » et les maires « développeurs »), tandis que les positions des particuliers et du Préfet varient selon que le contentieux administratif a lieu sur le littoral ou dans l'arrière-pays. Dans l'arrière-pays, le préfet apparaît développeur et les particuliers conservateurs; sur le littoral, leurs positions sont équilibrées entre les deux logiques.

Sur la politique de développement faisant l'objet de contestation, apparaissent des différences entre littoral et arrière-pays. Le préfet est le principal acteur public qui promeut une logique de développement d'activités, en l'occurrence essentiellement dans les zones de l'arrière-pays. Les décisions préfectorales litigieuses portent à cet égard sur des autorisations d'exploiter et des projets d'aménagement et d'infrastructure d'utilité publique. Plusieurs communes sont concernées par les premières, qui concernent des ICPE (Lunel-Viel ${ }^{10}$, Cazouls-lesBéziers ${ }^{11}$, Teyran ${ }^{12}$ ).

Des maires suivant une politique de développement d'activité et d'urbanisation appartiennent

10. Une association et plusieurs dizaines de particuliers (81) s'opposent à la décision du préfet de l'Hérault de délivrer à une entreprise un permis de construire portant sur la réalisation d'un centre de traitement et de valorisation de résidus urbains à Lunel-Viel (CAA Marseille, 17 septembre 1996).

11. Deux conflits concernent cette commune : une autorisation préfectorale d'exploitation d'une carrière de calcaire (CAA Marseille 8 septembre 1994, action d'un particulier); l'autorisation donnée par le préfet de l'Hérault à la communauté d'agglomération de Montpellier à exploiter jusqu'au 31 décembre 2006 le centre de stockage de déchets ménagers des jardins de Maguelone (CAA Marseille, 31 décembre 2002). Dans ce dernier cas, l'opposition est portée par l'association pour la défense de la nature et de l'environnement Maguelone-Gardiole.

12. Un résident demande l'annulation de l'arrêté du préfet autorisant une entreprise à exploiter une installation de concassage-criblage de matériaux de carrière (CE, 26 décembre 1983). 
également majoritairement à des communes de l'arrière-pays. Des décisions contestées d'urbanisation et de promotion immobilière sont en effet prises en grande majorité par les maires et conseils municipaux de communes intérieures (Lunel, Montferrier-sur-Lez, Saint-André de Buèges ${ }^{13}$, Saint-Bauzille de Putois ${ }^{14}$ ). Les deux seules communes littorales recensées sont La Grande-Motte et Palavas-les-Flots ${ }^{15}$. Les décisions allant dans le sens de l'implantation d'activités économiques ne concernent pas non plus les communes littorales (Fabrègues ${ }^{16}$, Saint-Mathieu de Tréviers ${ }^{17}$, Boujansur-Libron ${ }^{18}$ ). En revanche, les décisions liées à des équipements sont plus sensibles sur le littoral que dans les communes de l'intérieur. La région de Sète est à cet égard particulièrement sensible. Les deux principaux conflits sétois font l'objet de plusieurs décisions de justice, mettent en jeu l'action d'associations de protection de l'environnement. Le premier conflit porte sur la déclaration préfectorale d'utilité publique pour la construction d'une déviation du chemin départemental $n^{\circ} 2$ au Nord de Sète (entre la pointe longue et la pointe du Barrou ${ }^{19}$ ). Le second porte sur l'autorisation donnée à la commune de Sète par le préfet de réaliser des travaux d'aménagement relatifs au traitement et à l'évacuation des eaux pluviales de la ZAC du triangle de Villeroy $^{20}$. Mais dans ce cas, l'origine du contentieux

13. Préemption par la commune de parcelles pour lotir, contrecarrée par le propriétaire foncier.

14. Délivrance d'un permis de construire à un particulier, contestée par un voisin de la future construction (CE référé 27 juillet 2001).

15. À La Grande-Motte, la commune et une société civile immobilière dans le domaine hôtelier contestent l'annulation par le tribunal administration d'un permis de construire portant sur un ensemble hôtelier, annulation obtenue par une association de copropriétaires voisins (CE, 13 février 86). À Palavas-les-Flots, les copropriétaires d'un lotissement agissent contre le permis de construire délivré à un promoteur (CAA Bordeaux, 4 juillet 1990). Dans un autre cas, une association (le Comité de liaison pour la vie des étangs montpelliérains) forme un recours contre l'arrêté du maire de Palavas-les-Flots accordant un permis de lotir un promoteur immobilier (CAA Bordeaux, 7 avril 1993).

16. Modification du Plan Local d'Urbanisme en vue de l'installation d'un centre de traitement des déchets SITA-Sud (CAA Marseille, référé, 30 mars 2005)

17. Contestation par le «Collectif de réflexion sur le Plan d'Occupation des Sols de Saint-Mathieu-de-Tréviers » de l'arrêté du maire accordant à une entreprise un permis de construire une centrale à béton $(\mathrm{CE}$, 10 février 1987).

18. L'association syndicale du lotissement Monestié conteste la décision du maire d'autoriser une entreprise à construire divers bâtiments à usage de centrale à béton sur la zone d'activité économique de la zone d'aménagement concerté du Monestié (CE, 4 février 1987).

19. CE, Société de protection de la nature de Sète-Frontignan-Balaruc, 13 mai 1988.

20. CE, Association entre mer et étang Sète Environnement, 27 novembre 2003. est dans les faits liée à l'urbanisation controversée de ce nouveau quartier sétois...

Enfin, la structure des oppositions dans le contentieux administratif apparaît différenciée entre communes littorales et communes intérieures. Les associations de protection de l'environnement sont plus majoritairement demandeurs (c'est-à-dire requérants) dans les communes littorales que dans les communes intérieures, où les particuliers sont plus fréquemment à l'origine de l'action dans le contentieux administratif (figure 7). Les communes sont, dans la zone littorale, plus fréquemment défendeur que dans l'intérieur du département. Dans toutes les communes, littorales et intérieures, ce sont les pouvoirs locaux, Etat et communes, qui font l'objet de contestations de la part de la société civile, c'està-dire les particuliers et les associations de protection de l'environnement qui sont par ailleurs pour l'essentiel des associations de riverains.

\section{Discussion et ConcLusions}

L'objectif de cette étude était de démontrer la spécificité des conflits « littoraux » par rapport à ceux de « l'intérieur » à partir de l'analyse des jugements de second degré rendus par les juridictions du département de l'Hérault. La démonstration en a été faite à partir de plusieurs approches. Une première approche, essentiellement quantitative, a permis de faire ressortir que la surreprésentation de la part du contentieux, notamment ceux qui sont liés à l'urbanisme, dans les communes littorales. Une seconde approche, couplant analyse géographique et exploitation d'analyses factorielles, a révélé que les requêtes contentieuses sur le littoral étaient principalement liées à des enjeux de protection de l'environnement naturel, contrairement aux conflits de l'arrière-pays, plus liés à la régulation des nuisances dues à l'urbanisation. Enfin, une troisième et dernière approche, dédiée à l'analyse des logiques d'acteurs sous-jacentes aux conflits, montre que, dans les communes littorales, les requêtes en appel sont portées devant le juge administratif dans une logique de protection du patrimoine naturel, essentiellement par des particuliers et des associations locales. Dans les communes de l'arrière-pays, les requêtes sont déposées par les particuliers et les élus locaux (et dans une moindre mesure les entreprises) dans une logique de développement économique et urbain. 


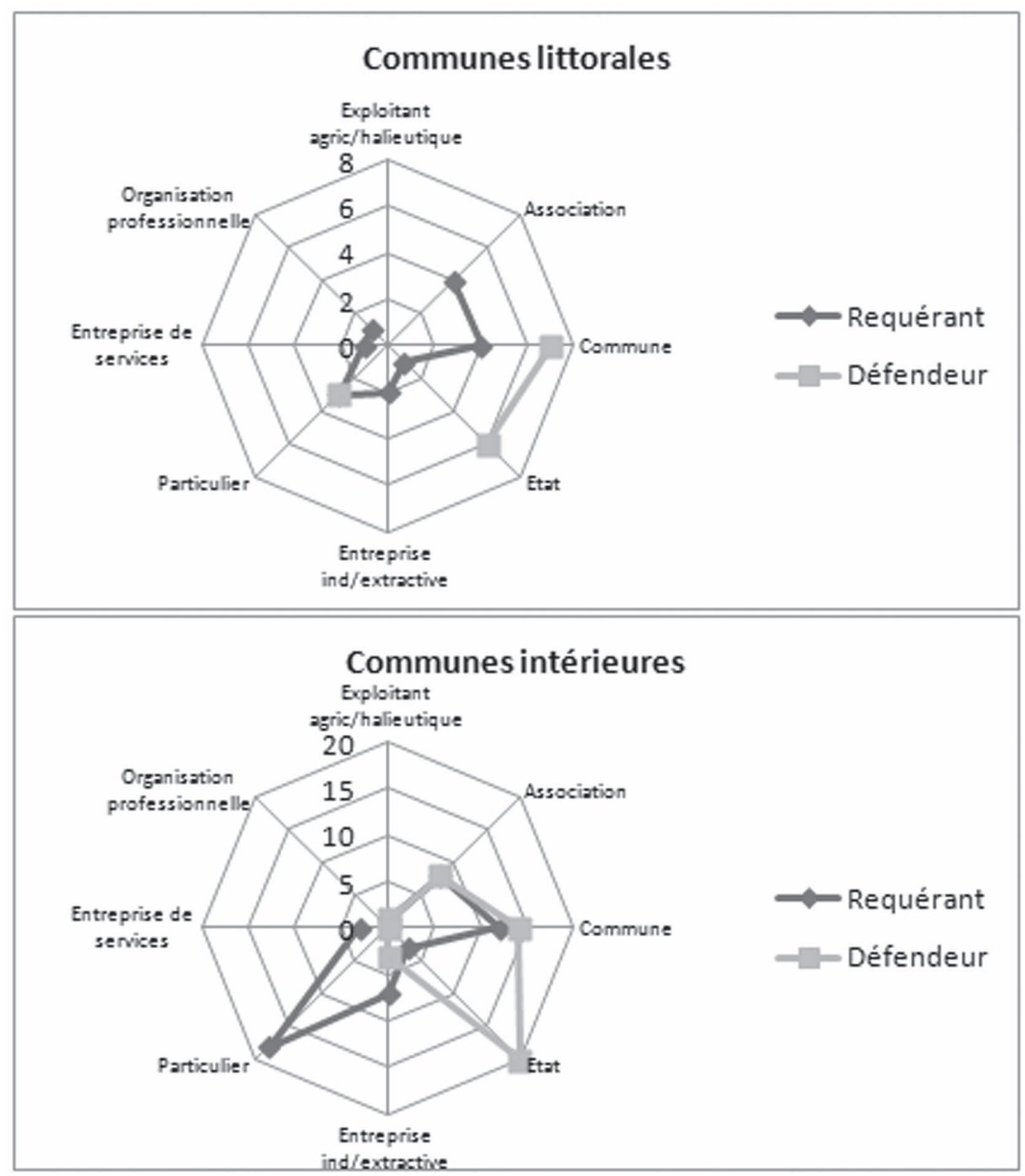

Figure 7 : Acteurs du contentieux administratif de deuxième instance (requérants et défendeurs, nombre de conflits) Actors of administrative appeal litigation (plaintiff and defendor)

Par ailleurs, les conflits littoraux se distinguent aussi par le fait que les enjeux en termes d'aménagement et de gestion mobilisent différemment les acteurs publics, et notamment au niveau de l'État. Autant les maires apparaissent dans une logique de développement systématique, sur le littoral comme dans l'arrière-pays, autant le positionnement du Préfet apparaît plus nuancé, entre conservation et développement, sur le littoral que dans l'arrière-pays où il soutient majoritairement le développement. Dans un contexte de décentralisation, les actions de justice administrative du deuxième niveau montrent que l'Etat représente un possible garant de la conservation des milieux. En effet, sur un espace restreint, fragile, où la préoccupation environnementale est forte et disposant d'une réglementation spécifique
(Loi Littoral), les acteurs publics sont confrontés à des enjeux majeurs en termes d'articulation d'usages et d'intérêts d'acteurs agissant dans des secteurs spécifiques (pêche, tourisme, transport...), d'articulation des documents de planification et d'articulation des initiatives entre les échelles de gestion (de l'échelle internationale à l'échelle micro-locale). La « gestion intégrée » des zones côtières, souhaitée par les organisations internationales et nationales depuis les années 1990, constitue un cadre d'action publique spécifique qui explique le positionnement différencié de l'Etat par rapport à l'intérieur.

Enfin, l'analyse du contentieux de seconde instance donne à voir l'importance des questions d'urbanisme dans les dynamiques d'opposition sur le littoral. Elle rejoint en cela les résultats de l'analyse 
de la presse quotidienne régionale, sachant que les entretiens réalisés sur ce terrain vont également dans ce sens (Cadoret et Lavaud-Letilleul, 2011). En effet, au milieu des années 2000, sur le littoral héraultais, les conflits liés à l'urbanisme sont les plus souvent cités dans la presse, car c'est bien la remise en cause de la gestion et de la préservation du milieu par les opérations d'urbanisme qui est le plus fréquemment à l'origine des conflits.

Pour finir, cette analyse comporte deux limites qui appellent de nouvelles pistes de recherche. Premièrement, comme cela a été dit en première partie, il existe une grande diversité de conflits que ne met pas, ou peu, en évidence l'étude du contentieux de seconde instance, tels que les oppositions concernant le traitement des déchets, la gestion et la qualité de l'eau ou encore la cohabitation d'activités sur les espaces lagunaires. Ces affaires ne sont en effet pas toujours portées devant les juges ou, si elles le sont, ne font pas systématiquement l'objet d'appel. Il importerait donc pour affiner cette analyse des conflits à partir des sources juridiques de regarder sur un territoire plus restreint, notamment littoral, la complexité des cas portés devant les tribunaux, y compris de première instance.

Deuxièmement, l'étude du contentieux de seconde instance dans le département de l'Hérault a montré la spécificité des conflits littoraux par rapport aux conflits intérieurs.

Or la spécificité des conflits littoraux par rapport aux conflits intérieurs, ainsi démontrée, ne doit selon nous pas être interprétée comme le signe d'une indépendance stricte entre l'espace littoral et l'espace de l'arrière-pays. Certes, les conflits litto- raux témoignent de l'impact d'un contexte politique et réglementaire spécifique, dont l'objectif est d'assurer la non-urbanisation des espaces littoraux, d'en préserver l'accès et de préserver des écosystèmes naturels (dans le cas du littoral héraultais, les « coupures vertes » aménagées par la Mission Racine y ont de fait préservé des espaces naturels). Ce cadre institutionnel, dont se saisissent les associations et particuliers pour défendre la conservation d'un cadre de vie résidentiel à dimension environnementale devant les juges administratifs, accentue dans les faits la rareté du foncier urbanisable, ouvert à l'implantation d'activités économiques ou d'infrastructures d'utilité publique. Du coup, ces dernières ne tendent-elles pas à se redéployer à l'intérieur des terres, sur des espaces qui connaissent euxmêmes une importante activité résidentielle, là où les arguments de préservation des sites et milieux remarquables du littoral ne peuvent être mobilisés? Il serait de ce point de vue intéressant de voir dans des recherches futures si, bien que distincts dans leur objet et leur logique d'action, les conflits littoraux ne font pas système avec les conflits intérieurs, auxquels ils seraient alors liés par des dynamiques territoriales se déployant à l'échelle du département.

\section{Remerciements}

Cet article est issu de projets collectifs et pluridisciplinaires soutenus par l'ANR (ANDYCOTE) et le programme Agriculture et Développement Durable (COVER).

\section{Annexe 1 - Variables utilisées dans l'analyse de données (entre parenthèses : effectifs)}

\begin{tabular}{|l|l|l|l|l|}
\hline LIBELLÉ & Description & Modalités & source & $\begin{array}{l}\text { Type de variable } \\
\text { (ACM) }\end{array}$ \\
\hline Comm_lit & Commune littorale ou intérieur & $\begin{array}{l}1 \text { littoral (15) } \\
2 \text { non littoral ( 41) }\end{array}$ & Source : INSEE & Nominale active \\
\hline obj_acces & $\begin{array}{l}\text { Objet du conflit : accessibili- } \\
\text { tés, servitudes, autres actions } \\
\text { possessoires }\end{array}$ & $\begin{array}{l}1 \text { objet acces (13) } \\
2 \text { no objet acces (43) }\end{array}$ & $\begin{array}{l}\text { Base de données Conflits } \\
\text { d'usage (auteurs) }\end{array}$ & Nominale active \\
\hline obj_agric & $\begin{array}{l}\text { Objet du conflit : agriculture / } \\
\text { halieutique / foresterie }\end{array}$ & $\begin{array}{l}1 \text { obj agri oui (2) } \\
2 \text { no obj agri (54) }\end{array}$ & $\begin{array}{l}\text { Base de données Conflits } \\
\text { d'usage (auteurs) }\end{array}$ & Nominale active \\
\hline obj_icpe & $\begin{array}{l}\text { Objet du conflit : activité indus- } \\
\text { trielle ou extractive soumise à } \\
\text { autorisation (ICPE) }\end{array}$ & $\begin{array}{l}\text { 1 obj icpe oui (10) } \\
2 \text { no obj icpe (46) }\end{array}$ & $\begin{array}{l}\text { Base de données Conflits } \\
\text { d'usage (auteurs) }\end{array}$ & Nominale active \\
\hline
\end{tabular}




\begin{tabular}{|c|c|c|c|c|}
\hline obj_ind & $\begin{array}{l}\text { Objet du conflit : activité indus- } \\
\text { trielle ou extractive non soumise } \\
\text { à autorisation }\end{array}$ & $\begin{array}{l}1 \text { obj ind oui }(2) \\
2 \text { no obj ind }(54)\end{array}$ & $\begin{array}{l}\text { Base de données Conflits } \\
\text { d'usage (auteurs) }\end{array}$ & Nominale active \\
\hline obj_iup & $\begin{array}{l}\text { Objet du conflit : infrastructures } \\
\text { d'utilité publique }\end{array}$ & $\begin{array}{l}1 \text { obj iup oui (11) } \\
2 \text { no obj iup (45) }\end{array}$ & $\begin{array}{l}\text { Base de données Conflits } \\
\text { d'usage (auteurs) }\end{array}$ & Nominale active \\
\hline obj_preser & $\begin{array}{l}\text { Objet du conflit : gestion et } \\
\text { préservation milieu naturel }\end{array}$ & $\begin{array}{l}1 \text { obj preser oui (14) } \\
2 \text { no obj preser }(42)\end{array}$ & $\begin{array}{l}\text { Base de données Conflits } \\
\text { d'usage (auteurs) }\end{array}$ & Nominale active \\
\hline obj_urba & $\begin{array}{l}\text { Objet du conflit : opération ou } \\
\text { document d'urbanisme et amé- } \\
\text { nagement }\end{array}$ & $\begin{array}{l}1 \text { obj urba oui (18) } \\
2 \text { no obj urba }(38)\end{array}$ & $\begin{array}{l}\text { Base de données Conflits } \\
\text { d'usage (auteurs) }\end{array}$ & Nominale active \\
\hline obj_voisin & $\begin{array}{l}\text { Objet du conflit : voisinage } \\
\text { (règlement de co-propriété, } \\
\text { troubles de voisinage, vols et } \\
\text { dégradations) }\end{array}$ & $\begin{array}{l}1 \text { obj voisin oui }(7) \\
2 \text { no obj voisin }(49)\end{array}$ & $\begin{array}{l}\text { Base de données Conflits } \\
\text { d'usage (auteurs) }\end{array}$ & Nominale active \\
\hline dr_urbos & $\begin{array}{l}\text { Domaine juridique de la re- } \\
\text { quête : décisions et documents } \\
\text { d'urbanisme }\end{array}$ & $\begin{array}{l}1 \mathrm{dr} \text { urbos oui (36) } \\
2 \text { no dr urbos }(20)\end{array}$ & $\begin{array}{l}\text { Base de données Conflits } \\
\text { d'usage (auteurs) }\end{array}$ & Nominale active \\
\hline dr-iuep & $\begin{array}{l}\text { Domaine juridique de la } \\
\text { requête : infrastructures et équi- } \\
\text { pements utilite publique }\end{array}$ & $\begin{array}{l}1 \text { dr iuep oui (8) } \\
2 \text { no dr iuep (48) }\end{array}$ & $\begin{array}{l}\text { Base de données Conflits } \\
\text { d'usage (auteurs) }\end{array}$ & Nominale active \\
\hline dr_autoregul & $\begin{array}{l}\text { Domaine juridique de la } \\
\text { requête : activité réglementée } \\
\text { (régime autorisation) }\end{array}$ & $\begin{array}{l}1 \mathrm{dr} \text { autoreg oui (10) } \\
2 \text { no dr autoreg } 46)\end{array}$ & $\begin{array}{l}\text { Base de données Conflits } \\
\text { d'usage (auteurs) }\end{array}$ & Nominale active \\
\hline dr_regulreg & $\begin{array}{l}\text { Domaine juridique de la } \\
\text { requête : activité reglementée } \\
\text { (régulation) }\end{array}$ & $\begin{array}{l}1 \text { dr regulreg oui }(3) \\
2 \text { no dr regulreg }(53)\end{array}$ & $\begin{array}{l}\text { Base de données Conflits } \\
\text { d'usage (auteurs) }\end{array}$ & Nominale active \\
\hline dr_regulpratiq & $\begin{array}{l}\text { Domaine juridique de la re- } \\
\text { quête : pratiques réglementées }\end{array}$ & $\begin{array}{l}1 \text { dr regul prat oui }(1) \\
2 \text { no dr regul prat }(55)\end{array}$ & $\begin{array}{l}\text { Base de données Conflits } \\
\text { d'usage (auteurs) }\end{array}$ & Nominale active \\
\hline dr_indiv & $\begin{array}{l}\text { Domaine juridique de la re- } \\
\text { quête : actions individuelles }\end{array}$ & $\begin{array}{l}1 \text { dr indiv oui (20) } \\
2 \text { no dr indiv (36) }\end{array}$ & $\begin{array}{l}\text { Base de données Conflits } \\
\text { d'usage (auteurs) }\end{array}$ & Nominale active \\
\hline dyn_urba & $\begin{array}{l}\text { Nombre annuel moyen d'auto- } \\
\text { risations de construction } 1990 \\
\text { à } 2004\end{array}$ & $\begin{array}{l}<50 \text { auto const an }(33) \\
>50 \text { auto const an }(23)\end{array}$ & sitadel (MEDDTL) & Nominale active \\
\hline Aire urbaine & & $\begin{array}{l}\text { Aire Urb (39) } \\
\text { No Aire Urb (17) }\end{array}$ & INSEE & Nominale active \\
\hline $\begin{array}{l}\text { super_com- } \\
\text { mune }\end{array}$ & $\begin{array}{l}\text { superficie (ha) des } 4 \text { grands } \\
\text { types d'occupation du sol }\end{array}$ & (CONTINUE) & IFEN-Corine Coverland & $\begin{array}{l}\text { Continue illustra- } \\
\text { tive }\end{array}$ \\
\hline super_prot $\%$ & & (CONTINUE) & IFEN-Corine Coverland & $\begin{array}{l}\text { Continue illustra- } \\
\text { tive }\end{array}$ \\
\hline natura $2000 \%$ & & (CONTINUE) & IFEN-Corine Coverland & $\begin{array}{l}\text { Continue illustra- } \\
\text { tive }\end{array}$ \\
\hline znieff & & (CONTINUE) & IFEN-Corine Coverland & $\begin{array}{l}\text { Continue illustra- } \\
\text { tive }\end{array}$ \\
\hline sol_artific \% & & (CONTINUE) & IFEN-Corine Coverland & $\begin{array}{l}\text { Continue illustra- } \\
\text { tive }\end{array}$ \\
\hline sol_agric $\%$ & & (CONTINUE) & IFEN-Corine Coverland & $\begin{array}{l}\text { Continue illustra- } \\
\text { tive }\end{array}$ \\
\hline sol_foret $\%$ & & (CONTINUE) & IFEN-Corine Coverland & $\begin{array}{l}\text { Continue illustra- } \\
\text { tive }\end{array}$ \\
\hline sol_humide \% & & (CONTINUE) & IFEN-Corine Coverland & $\begin{array}{l}\text { Continue illustra- } \\
\text { tive }\end{array}$ \\
\hline nic & $\begin{array}{l}\text { Nombre d'établissements rele- } \\
\text { vant du régime des installations } \\
\text { classées }\end{array}$ & (CONTINUE) & $\begin{array}{l}\text { MEDDTL - base de données } \\
\text { Installations classées }\end{array}$ & $\begin{array}{l}\text { Continue illustra- } \\
\text { tive }\end{array}$ \\
\hline nseveso & $\begin{array}{l}\text { Nombre d'établissements Seveso } \\
\text { en } 2006\end{array}$ & (CONTINUE) & IFEN-Corine Coverland & $\begin{array}{l}\text { Continue illustra- } \\
\text { tive }\end{array}$ \\
\hline
\end{tabular}




\begin{tabular}{|c|c|c|c|c|}
\hline varpop & \begin{tabular}{|l} 
Evolution de la population \\
entre 1968 et 2006 (base 100 \\
1968)
\end{tabular} & (CONTINUE) & IFEN-Corine Coverland & $\begin{array}{l}\text { Continue illustra- } \\
\text { tive }\end{array}$ \\
\hline soldemigra & $\begin{array}{l}\text { solde migratoire annuel, 1999- } \\
2006\end{array}$ & (CONTINUE) & IFEN-Corine Coverland & $\begin{array}{l}\text { Continue illustra- } \\
\text { tive }\end{array}$ \\
\hline soldenat & solde naturel annuel, 1999-2006 & (CONTINUE) & IFEN-Corine Coverland & $\begin{array}{l}\text { Continue illustra- } \\
\text { tive }\end{array}$ \\
\hline pop_total & total population (1999) & (CONTINUE) & IFEN-Corine Coverland & $\begin{array}{l}\text { Continue illustra- } \\
\text { tive }\end{array}$ \\
\hline рор $<20 \%$ & $\begin{array}{l}\text { idem en } \% \text { de la population } \\
\text { totale de la commune }\end{array}$ & (CONTINUE) & IFEN-Corine Coverland & $\begin{array}{l}\text { Continue illustra- } \\
\text { tive }\end{array}$ \\
\hline pop20-39\% & $\begin{array}{l}\text { idem en } \% \text { de la population } \\
\text { totale de la commune }\end{array}$ & (CONTINUE) & IFEN-Corine Coverland & $\begin{array}{l}\text { Continue illustra- } \\
\text { tive }\end{array}$ \\
\hline pop40-59\% & $\begin{array}{l}\text { idem en } \% \text { de la population } \\
\text { totale de la commune }\end{array}$ & (CONTINUE) & IFEN-Corine Coverland & $\begin{array}{l}\text { Continue illustra- } \\
\text { tive }\end{array}$ \\
\hline pop60-74\% & $\begin{array}{l}\text { idem en } \% \text { de la population } \\
\text { totale de la commune }\end{array}$ & (CONTINUE) & IFEN-Corine Coverland & $\begin{array}{l}\text { Continue illustra- } \\
\text { tive }\end{array}$ \\
\hline рор $>75 \%$ & $\begin{array}{l}\text { idem en } \% \text { de la population } \\
\text { totale de la commune }\end{array}$ & (CONTINUE) & IFEN-Corine Coverland & $\begin{array}{l}\text { Continue illustra- } \\
\text { tive }\end{array}$ \\
\hline Nbre conflits & & (CONTINUE) & $\begin{array}{l}\text { Base de données Conflits } \\
\text { d'usage (auteurs) }\end{array}$ & $\begin{array}{l}\text { Continue illustra- } \\
\text { tive }\end{array}$ \\
\hline $\begin{array}{l}\text { Nbre cont- } \\
\text { adm }\end{array}$ & & (CONTINUE) & $\begin{array}{l}\text { Base de données Conflits } \\
\text { d'usage (auteurs) }\end{array}$ & $\begin{array}{l}\text { Continue illustra- } \\
\text { tive }\end{array}$ \\
\hline $\begin{array}{l}\text { Nbre cont- } \\
\text { judic }\end{array}$ & & (CONTINUE) & $\begin{array}{l}\text { Base de données Conflits } \\
\text { d'usage (auteurs) }\end{array}$ & $\begin{array}{l}\text { Continue illustra- } \\
\text { tive }\end{array}$ \\
\hline $\begin{array}{l}\text { Niveau } \\
\text { vieillissement }\end{array}$ & $\begin{array}{l}\text { Indice de vieillissement (popula- } \\
\text { tion de la commune) }\end{array}$ & (CONTINUE) & IFEN-Corine Coverland & $\begin{array}{l}\text { Continue illustra- } \\
\text { tive }\end{array}$ \\
\hline $\begin{array}{l}\text { Niv Revenus } \\
\text { moyens Com- } \\
\text { mune }\end{array}$ & $\begin{array}{l}\text { Revenu moyen des foyers fiscaux } \\
\text { en } 2001\end{array}$ & $\begin{array}{l}\text { total des revenus } \\
\text { fiscaux de la commune } \\
\text { / nombre des foyers } \\
\text { fiscaux de la commune, } \\
\text { données de la DGI en } \\
2001\end{array}$ & MINEFI-DGI & $\begin{array}{l}\text { Continue illustra- } \\
\text { tive }\end{array}$ \\
\hline
\end{tabular}

\section{Bibliographie}

Cadène P., 1990. L'usage des espaces périurbains, une géographie régionale des conflits, Espace Rural, n 118-119, p. $235-267$.

Cadoret A., 2011. Analyse des processus conflictuels : le cas du littoral du Languedoc-Roussillon, L'Espace Géographique, $\mathrm{n}^{\circ} 3$, p. 231-244.

Cadoret A., Lavaud-Letilleul V., 2011. Conflictualité sur le littoral héraultais d'après la presse quotidienne régionale, in Colloque Conflits et territoires: conflits d'usage et de voisinage dans les espaces ruraux et périurbains: quand les conflits durent..., Paris, 26 avril 2011. Séquence à 02:07:00 sur [http://podcast.agroparistech.fr/groups/agroparistech/ weblog/921fd/SAD_APT_Conflits_et_territoires.html].

Cadoret A., 2006. Conflits d'usage liés à l'environnement et réseaux sociaux : enjeux d'une gestion intégrée? Le cas du littoral du Languedoc-Roussillon, thèse de doctorat en géo- graphie-aménagement, Université Paul Valéry, Montpellier III, $586 \mathrm{p}$.

Cadoret A., Foulquier E., Guineberteau T., Lamberts C., Trouillet B., 2008. L'évaluation du phénomène conflictuel des territoires: un enjeu méthodologique, un enjeu pour l'action publique territoriale. Réflexions et expériences sur des espaces littoraux et maritimes, colloque de l'Association de Science Régionale De Langue Française «Territoires et action publique territoriale : nouvelles ressources pour le développement régional », Rimouski, 25-27 août 2008.

Caron A., Torre A., 2002. Les Conflits d'usages dans les espaces ruraux : une analyse économique, Sciences de la Société, vol. 57, p. 95-113.

Charlier B., 1999. La défense de l'environnement : entre espace et territoire: géographie des conflits environnementaux déclenchés en France depuis 1974, Thèse de géographie et aménagement, Université de Pau, 753 p. 
DARLY S., 2008. La spatialité des conflits d'usage au sein des zones périurbaines en Île-de-France, Norois, n² 209, p. 127-146.

DARLY S., 2009. Faire coexister ville et agriculture au sein des territoires périurbains. Antagonismes localisés et dynamiques régionales de la conflictualité. Analyse du cas de l'Ile-deFrance, thèse de doctorat en géographie, Ecole des Hautes Etudes en Sciences Sociales, 480 p.

Donadieu P., Fleury A., 2003. La construction contemporaine de la ville-campagne, Revue de géographie alpine, n 91, 4, p. 19-28.

Goldberg E.-D., 1994, Coastal zone space : prelude to conflict?, La Jolla, Unesco, 138 p.

INSEE, 2009. La campagne et les villes-centres de plus en plus attractives en Languedoc-Roussillon, Repères synthèse pour l'économie du Languedoc-Roussillon, $\mathrm{n}^{\circ}$ 1, 5 p.

KAYSER B., 1989. La renaissance rurale. Sociologie des campagnes du monde occidental, Paris, Armand Colin, 316 p.

Kirat T., Lefranc C., 2004. Les conflits d'usage et leur expression territoriale: une analyse des profils contentieux de sept départements français, $4^{\text {th }}$ Proximity Congress : Proximity, Networks and Co-ordination, Marseille, 17-18 juin 2004. [http://halshs.archives-ouvertes.fr/halshs-00004203].

Kirat T., Torre A. (dir.), 2006. Conflits d'usages et dynamiques spatiales. Les antagonismes dans l'occupation des espaces périurbains et ruraux, dossier thématique de la revue Géographie, économie et société, vol. 8 (2006/3), 120 p.

Kirat T., Torre A. (dir.), 2007. Conflits d'usages et dynamiques spatiales. Les antagonismes dans l'occupation des espaces périurbains et ruraux, dossier thématique de la revue Géographie, économie et société, vol. 9 (2007/2), 110 p.

Lascoumes P., 1994. L'éco-pouvoir, environnements et politiques, Paris, La Découverte, 320 p.

Lascoumes P., Timbart O., 1993. La protection de l'environnement devant les tribunaux judiciaires répressifs, Infostat Justice, $n^{\circ} 34,4$ p.

Lavaud-Letilleul V., Cadoret A., L'urbanisation illégale sur le littoral du Languedoc-Roussillon : entre laisser-faire et reprise en main par les pouvoirs publics, dans Perrin C. (dir.), La Méditerranée : vers un littoral sans nature? Les espaces agricoles et naturels face à l'urbanisation, École française de Rome (sous presse).

Le Caro Y., 2002. Usages récréatifs de l'espace agricole, Thèse de géographie, Université de Rennes 2, 779 p.

Leost R., 1998. La stratégie contentieuse d'une association de protection de la nature en Bretagne, dans Le Louarn P. (dir.), Décision et droit de l'environnement, étude comparée des cas breton et martiniquais, Rennes, PUR, p. 85-106.

Le Roy Ladurie E., 1962. Histoire du Languedoc, coll. "Que sais-je? », n 958, Paris, PUF, 128 p.

Ley D., Mercer J., 1980. Locational conflict and the politics of consumption, Economic geography, vol. 56, n² 2, p. 89-109.

MÉLÉ P., 2004. Conflits et controverses : de nouvelles scènes de production territoriale? Colloque Espaces et sociétés aujourd'hui. La géographie sociale dans les sciences sociales et dans l'action, Rennes, 21-22 octobre 2004, 12 p.

Melot R., Pelisse J., 2008. Prendre la mesure du droit. Enjeux de l'observation statistique pour la sociologie juridique, Droit et société, vol. 69-70, p. 331-346.

NikolaïDou S., 2009. L'urbanisation littorale de la plaine viticole de Messoghia : entre villégiature et périurbanisation, Communication aux rencontres thématiques internationales Typologies des conflits littoraux, École Française de Rome, Madrid, 3 et 4 juillet 2009 [http://www.efrome.it/fr/PDF/ CVZURBA...].

Paoli J.-C., Melot R., Fiori A., 2008. L'aménagement du littoral à l'épreuve de la décentralisation, Pôle Sud: Revue de science politique de l'Europe méridionale, n²2, p. 143-165.

Рнam H.-V., Kirat T., 2008. Les conflits d'usage des espaces périurbains et le contentieux administratif. Le cas de la région Ile-de-France, Revue d'économie régionale et urbaine, $\mathrm{n}^{\circ} 8$, p. 671-700.

Racine P., 1980. Mission impossible? : l'aménagement touristique du littoral du Languedoc-Roussillon, Montpellier, Editions Midi libre, coll. « Témoignages », 293 p.

Renaud Y., 2001. De la contestation à la concertation, Les Annales de la recherche urbaine, $\mathrm{n}^{\circ} 89$, p. 62-69.

Rialland C., 2003. Les conflits d'usage aux limites de la ville, dans Mélé P., Larrue C., Rosemberg M. (Coord.), Conflits et Territoires, Tours, PUFR, MSH «Villes et Territoires ", p. 189-204.

Rouzier J. (dir.), 2002. Le Languedoc-Roussillon 1950-2001: histoire d'une mutation, Toulouse, Editions Privat, 236 p.

SÉnÉCAL G., 2002. Controverses en environnement et développement durable urbain : la formation d'un nouvel espace public. Dossier : La ville et l'environnement, VertigO, vol. 3, $\mathrm{n}^{\circ} 2$, p. 2-6. [http://vertigo.revues.org/3762]; DOI : 10.4000/ vertigo. 3762 .

Torre A., Melot R., Bossuet L., Cadoret A., Caron A., Jeanneaux P., Kirat T., Pham H.-V., 2010. Comment évaluer et mesurer la conflictualité liée aux usages de l'espace? Éléments de méthode et de repérage, VertigO-La revue des sciences de l'environnement, vol. 10, $\mathrm{n}^{\circ} 1, \mathrm{p} .1-26$. [http:// vertigo.revues.org/9590]; DOI : 10.4000/vertigo.9590.

Trudelle C., 2003. Au-delà des mouvements sociaux : une typologie relationnelle des conflits urbains, Cahiers de géographie du Québec, vol. 47, n 131, p. 223-242.

Vallega A., 1999. Fundamentals of integrated Coastal Management, The Geo Journal Library, Kluwer Academic Publishers, $262 \mathrm{p}$.

Villeneuve P., Côté G., 1994. Conflits de localisation et étalement urbain : y a-t-il un lien? Cahiers de Géographie du Québec, vol. 38, n 105, p. 397-412.

Villeneuve P., Trudelle C., Pelletier M., Thériault M., 2009. Les conflits urbains : une approche analytique, Environnement urbain/Urban Environment, vol. 3, p. 64-82. 